\title{
The disturbance effect of white noise on human short-term memory during learning
}

WALTER SLOBODA, Behavioral and Clinical Studies Research Center, Saint Elizabeths Hospital, Washington, D.C. 20032

The Ss had to learn a seven-digit number presented either with or without a 5-sec 72-dB burst of white noise. No significant difference was found in recall between white-noise and no-white-noise conditions with either a 2- or 12-sec retention interval (RI). GSR measures did not differentiate the white-noise and no-white-noise conditions, nor were differences found in recall performance between trials with large drops in resistance and those showing minimal changes. $A$ rho of -.86 was found for the 12-sec RI between background skin resistance (BSR) and performance, with those Ss having low $B S R$ performing more poorly than those Ss having highef BSR. No comparable correlation was found for the 2-sec RI.

Sloboda \& Smith (1968) found that the recall of seven-digit numbers after 2 or $12 \mathrm{sec}$ filled retention intervals (RIs) was unaffected by whether of not a $2-\sec$ burst of white noise occurred during the RI. Concomitant measures indicated, however, that, physiologically, the white noise did have some effect, i.e., GSR latency varied with white noise placement. The present study is an attempt to determine the effects of white noise on digit recall when it is presented with, rather than after, the to-be-remembered material.

\section{METHOD}

The Ss were 16 male and 16 female students enrolled at universities in the Washington, D. C. area; their ages ranged from 18 to 29 years, and they were paid for their participation.

Upon arrival at the laboratory, each $\mathrm{S}$ was first given two trials on a digit-span task to insure that $S$ was able to correctly recall seven digits in order when the interval and activity intervening between presentation and recall were minimized. Each seven-digit number was presented visually for 5 sec and the $S$ was required to recall the digits aloud in their presentation order as soon as their exposure terminated. All 32 Ss were able to perform this task perfectly on at least four of the seven trials. A pair of GSR electrodes were then attached to the $S$, and he was seated in a wooden chair. He was then given four trials on an auditory discrimination task. On each trial, two bursts of white noise of varying loudness were presented in succession and the $S$ was required to specify which was louder. The purpose of this task was to insure that an absence of a white-noise effect in the main experiment could not be attributed to the Ss' insensitivity to the intensity of the disturbing agent. All 32 Ss were able to discriminate differences of $10 \mathrm{~dB}$ or less. Taped instructions were then played to the $S$. The total time elapsing between attachment of the electrodes and the start of the experiment was $20 \mathrm{~min}$. During the rest of the experiment the $S$ worked alone in a semidarkened room.

One variable in this experiment was the duration of the RI, either 2 or $12 \mathrm{sec}$. For ease of exposition the procedure for the 12 -sec RI condition will be discussed first. Sixteen of the 32 Ss worked with a 12-sec RI, and each of these Ss was given two practice and 24 test trials. The components of a trial consisted of three slides. First, a seven-digit number was exposed for 5 sec and the $S$ was instructed to read the digits aloud from left to right. Second, six common words, each printed in upper case letters, were presented for $12 \mathrm{sec}$. The words were arranged in a vertical column and the $S$ was instructed to read each word aloud and then to indicate whether the word pertained to people or to objects by saying aloud either "people" or "objects." In addition to classifying each word, the $S$ was also required to start with the top word and proceed

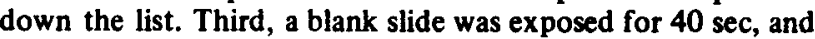
at the onset of this period the $S$ was cued (by a $1,000-\mathrm{Hz}$, $50-\mathrm{dB}$ tone) to attempt to recall the seven-digits in the order in which they had been presented. At the end of the 40 -sec period, the $S$ was again cued (by the same tone) that the next set of digits was about to appear.

The within-S variable was the presence or absence of a 5-sec burst of white noise during the digits. Two sets of 24 test trials (Sets $\mathrm{AB}$ and $\mathrm{CD}$ ) were used in the experiment. Each set of 24 test trials was divided into two blocks of 12 consecutive trials (Blocks $\mathrm{A}$ and $\mathrm{B}$, or Blocks $\mathrm{C}$ and $\mathrm{D}$ ), representing the two possible presentation orders of the experimental conditions. Before the start of each block of 12 trials, $\mathrm{S}$ was told whether or not white noise would accompany the digits. Eight Ss (four male and four female) were tested with Set $\mathrm{AB}$, and eight other Ss (four male, four female) with Set $C D$, thus resulting in a counterbalanced Latin square design.

The procedure for the 2-sec RI conditions was the same as that for the 12-sec conditions except that the $16 \mathrm{Ss}$ had to classify and give a word similar in meaning to only the top two words of the six-word list. The presentation orders and assignment of sets to conditions were identical to those used for the 12-sec RI.

The materials and appartus used in this experiment were the same as those described in detail by Sloboda \& Smith (1968). Briefly, the seven-digit numbers were constructed by randomly sampling seven digits, without replacement, from the digits 0 through 9. The words were selected from the high-frequency range of the Thorndike-Lorge (1944) frequency-of-occurrence norms. The GSR was obtained from a Fels Dermohmeter (Model 22A) using zinc-zinc sulfate paste and the standard Lucite cup electrodes. A six-channel Offner Type R Polygraph was used to record the GSR and the slide presentation. RESULTS AND DISCUSSION

The basic data obtained from the Ss on each trial was the total number of digits whose serial position in the S's recall response matched the serial position in the S's original reading of the digits. In Fig. 1 the mean per cent correctly recalled digits are plotted for the seven serial positions and both RIs. Analyses of variance were performed for the two RIs separately to test the effects of serial position, the presence or absence of white noise, and the interaction of these two variables. Significance was found for the effect of serial position under both RIs ( $F=23.81$, df $=6 / 90$, $p<.01$, for the $2-\sec \mathrm{RI} ; \mathrm{F}=4.31, \mathrm{df}=6 / 90, \mathrm{p}<.01$, for the $12-\mathrm{sec} \mathrm{RI})$, but neither for the white noise conditions nor for the interaction of white noise and serial position in either of the RIs.

In order to assess the effects of white noise on the GSRs, the largest drop in skin resistance beginning between 1 and 3 $\sec$ after the onset of the digit slide was determined. These GSRs were then converted into changes in log conductance. For the 2-sec and 12-sec RIs the presence of white noise did not significantly affect the amplitude of the GSRs. Thus, the 72-dB white noise influenced neither recall nor the GSR amplitudes. To investigate the possible effects of differential physiological levels on recall, all of the trials for each S were ranked on the basis of the GSR amplitude and the mean recall scores of the top and bottom quartiles of these ranks were determined. The average number of correctly recalled digits, 


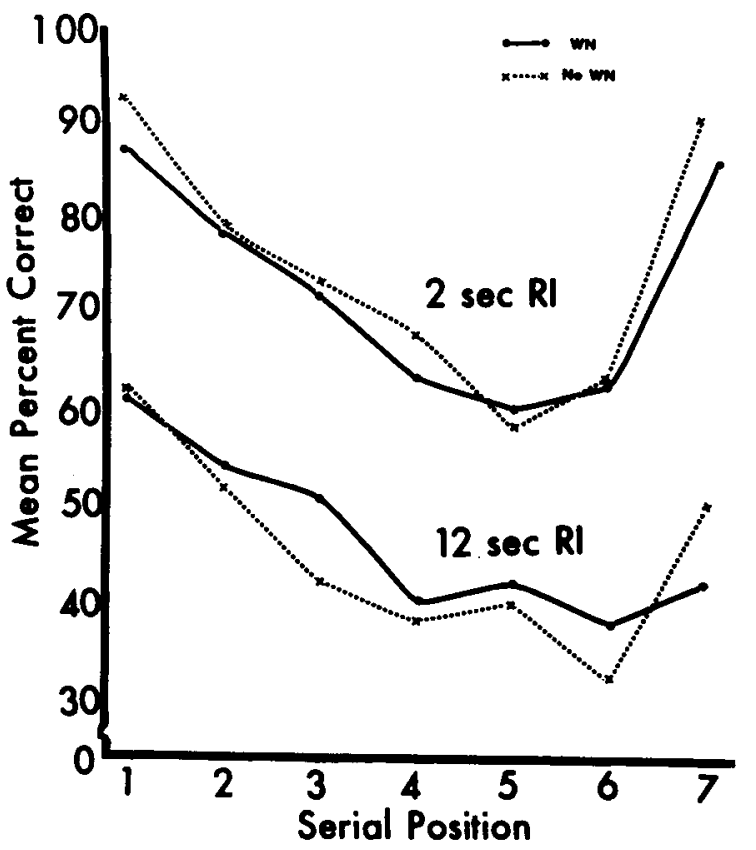

Fig. 1. Mean per cent correctly recalled digits as a function of serial position, white noise and retention intervals.

averaged over Ss, were 5.3 and 5.1 for the $2-\sec$ RI and 3.1 and 3.1 for the 12-sec RI for the top and bottom quartiles, respectively. Again, no disruption effect was found even with differing physiological responsiveness. Although Sloboda and Smith reported a physiological measure which correlated with the onset of white noise (latency of onset for the largest GSR during the 12-sec RI), no such evidence was found in the present study.

One possible explanation for a lack of differential physiological activity to the white noise-no white noise variation may be that the warning which Ss received prior to the beginning of the white noise condition eliminated the surprise or startle-value of the white noise and resulted in quick adaptation. ${ }^{1}$

The negative results reported both in this study and in that of Sloboda \& Smith (1968) suggest that 72-dB intensity white noise has little, if any, effect on short-term memory when rehearsal is minimized. These two studies taken together appear to indicate that $72-\mathrm{dB}$ white noise can neither influence a reverberation nor consolidation process.

In order to obtain some measure of interindividual performance an "arousal level" was computed for each S. This value was the average of the background skin resistance (BSR) in ohms at the onset of the white noise and no white noise blocks. The performance measure was the per cent of correctly recalled digits for the two blocks. Each of the 17 Ss was ranked on the basis of arousal level and per cent of correctly recalled digits. Higher ranks were given to higher BSR values and poorer performance. For the 12-sec interval, a Spearman tho correlation of $-.86(\mathrm{p}<.01)$ was found between the two sets of ranks, while for the 2-sec RI a nonsignificant tho of +.25 was obtained. The significant negative correlation which was obtained for the 12-sec RI indicates that Ss with lower BSR levels (i.e., those Ss showing greater arousal) performed more poorly than those with higher BSR levels (lower arousal) in this RI. When the RI was shortened to $2 \mathrm{sec}$ this inverse relationship between arousal level and performance did not hold.

Two explanations are suggested for these results. First, no significant correlation was found for the 2-sec RI because the task was too easy and did not differentiate performance sufficiently between the Ss. The second and perhaps more plausible explanation is that two memory systems may be involved, each with its own physiological indicators. For the 12-sec RI, performance appears to be a function of the tonic, stable arousal level of an organism. For the shorter, 2-sec RI, performance seems to be independent of the GSR level and is probably correlated with some more dynamic, shorter latency fluctuation in the physiological state of the organism.

Weiner (1966) has reported a series of studies which investigated the effects of motivation on memory by changing the quality and magnitude of incentive. Weiner concluded that "Stimuli a priori considered to be highly arousing because of their association with an affective consequence are more likely to be recalled after a relatively short time interval than stimuli considered to be relatively low in arousal value [p. 20]." Since no physiological measures of arousal were employed, further research using Weiner's methodology as well as concomitant physiological measures may provide a clearer picture of the relationship between arousal and memory.

\section{REFERENCES}

SLOBODA, W., \& SMITH, E. Disruption effects in human short-term memory: Some negative findings. Perceptual \& Motor Skills, 1968, 27, 575-582.

WEINER, B. Motivation and memory. Psychological Monographs: General \& Applied, 1966, 80 (Whole No. 626). NOTE

1. Sloboda \& Smith (1968) reported that in a pilot study, significant amplitude differences were found between white-noise/no-white-noise trials when they were interspersed. No differences in recall were found, however.

\section{DISCUSSION}

The effectiveness of anchor stimuli in modifying judgments of duration supported the main conjecture of this study. A tentative conclusion may be that Ss respond to attributes of stimuli which are commonly considered irrelevant to the response dimension, or, more generally, that the concept of relevance should be extended across stimulus dimensions as well as across modalities.

The direction of the effects was somewhat surprising, since, regardless of anchor intensity (excepting $A=S$ ), judgments of duration were lowered from the control function. A highly tentative explanation is that a pure tone has qualities which may make it more salient in perception than an interval of noise, presumably a more diffuse stimulus. An additional assumption, that perceived discrepancy from the series stimuli

\section{from page 81)}

enhances the distinctiveness of the anchor stimulus and, hence, its effectiveness, may account for the disparity between $A=S$ and the other anchor conditions.

Further work under consideration in this area will investigate the combined effects of anchor stimuli differing both in attributive qualities, as expressed in this paper, and in modality.

\section{REFERENCES}

KRUSKAL, W. H, \& WALLIS, W. A. Use of ranks in one-criterion variance analysis. Journal of the American Statistical Association, $1952,47,583-621$.

LONDON, I. D. Research on sensory interaction in the Soviet Union. Psychological Bulletin, 1954, 51, 531-568.

$$
\text { NOTE }
$$

1. This research was supported by the Air Force Office of Scientific Research, Grant No. 1163-66. 